\title{
Creencia global en un mundo justo: Validación de la escala de liPKUS en ESTUDIANTES UNIVERSITARIOS DE LA CIUDAD DE BUENOS AIRES
}

\section{GLobal belief In A JUST WORLD: VALIDATION OF THE LIPKUS SCALE IN UNIVERSITY STUDENTS FROM CITY OF BUENOS AIRES}

\author{
Alicia Barreiro*, Edgardo Etchezahar** Y Vicente Prado-Gascón ${ }^{* * *}$
}

\begin{abstract}
'Doctora en el área de Ciencias de la Educación, Magister en Psicología Educacional y Licenciada en Psicología. Miembro de la Carrera del Investigador Científico del Consejo Nacional de Investigaciones Científicas y Técnicas (CONICET) y Jefa de Trabajos Prácticos en la asignatura Psicología y Epistemología Genética I en la Facultad de Psicología de la Universidad de Buenos Aires (UBA).E-Mail: abarreiro@psi.uba.ar Gral. Juan Lavalle 2353 (C1052AAA), Ciudad Autónoma de Buenos Aires.

**Magister en Psicología Cognitiva y Aprendizaje y Licenciado en Psicología. Jefe de Trabajos Prácticos de la asignatura Psicología Política II de la Facultad de Psicología de la Universidad de Buenos Aires (UBA) y Becario

Tipo Il del Consejo Nacional de Investigaciones Científicas y Técnicas (CONICET).

E-Mail: edgardoetchezahar@psi.uba.ar

${ }^{* * *}$ Doctor en Psicología Social y de las Organizaciones y Licenciado en Psicología. Profesor de la Universidad Europea de Valencia. E-Mail: vicente.prado@uv.es
\end{abstract}

\section{RESUMEN}

El objetivo principal del trabajo que se informa fue adaptar y validar la Escala de Creencia Global en un Mundo Justo (Global Belief in a Just World Scale - GBJWS) de Lipkus (1991) en una muestra de estudiantes universitarios de la Ciudad de Buenos Aires (Argentina). Se administró un instrumento de evaluación a 328 estudiantes con edades entre 18 y 40 años $(M=24.1$; $D T=3.88)$, de los cuales el $36.6 \%$ eran hombres $(n=120)$ y el $63.4 \%$ mujeres $(n=208)$. Los resultados obtenidos sugieren adecuadas propiedades psicométricas de la escala GBJWS en la muestra con la que se trabajó. Los análisis realizados confirman la unidimensionalidad de la escala. Asimismo, las puntuaciones en la GBJWS se relacionan positivamente con el autoritarismo del ala de derecha (Right Wing Authoritarianism $R W A$ ), la orientación a la dominancia social (Social Dominance Orientation - SDO) y negativamente con el autoposicionamiento ideológico po- lítico. Se considera que el estudio realizado abre una línea de investigación que permite la indagación sistemática de la creencia global en un mundo justo en contextos hispanoparlantes a partir de la construcción de una medida confiable y válida para el contexto argentino. Se recomienda la realización de futuros estudios que utilicen la GBJW en poblaciones diferentes a la estudiada, para así avanzar con respecto a la generalización y representatividad de los resultados presentados en este trabajo.

Palabras clave: Lipkus; Creencia Global en un Mundo Justo; Argentina; Validación; Autoposicionamiento ideológico político; RWA; SDO; Estudiantes universitarios.

\section{ABSTRACT}

The researches on the Belief in a Just World (BJW) started in the 60' with the experimental 
studies carried out by Lerner (Lerner, 1965, 1980; Lerner \& Clayton, 2011; Lerner \& Simmons, 1966). Those studies pointed out that social injustices are frightening for individuals, therefore they need to belief that everyone gets what deserve during his or her life to maintain the control over their environment. If people do not believes that things happen as consequences of their acts, they will feel exposed to undesirable situations that today happen to others, but tomorrow could happen to them. Then, to deny this threat, they blame the victims of injustice, attributing to them the responsibility for their suffering. During the last decades, different studies have shown that individuals from different cultures around the world believe that the world is a just place, and the relations between this belief and socio-political factors (Furnham, 1993, 1998, 2003). In this vein, the system justification theory proposes that the BJW, together with the social dominance orientation (SDO; Jost \& Hunyady, 2005; Pratto, Sidanius, Stallworth \& Malle, 1994), contributes to support and rationalize the social order denying social injustices by appealing to the merit of the dominant social groups. Moreover, there are evidence that the BJW is positively associated to right wing authoritarianism ( $R W A$ - Altemeyer, 1996; Dalbert \& Yamauchi, 1994; Moore, 2008) and political ideology self positioning (PI Schlenker, Chambers \& Le, 2012). The aim of this paper is to adapt and validate a Spanish version of the Global Belief in a Just World Scale (GBJWS) by Lipkus (1991) in a sample of university students. Hence, a self-reported questionnaire was administered to 328 university students of the Autonomous City of Buenos Aires (Argentina) aged between 18 and 40 years old $(M=24.1$, $S D=3.88), 36.6 \%$ were male $(n=120)$ and $63.4 \%$ female $(n=208)$. Besides, $61.28 \%$ of the participants studied Psychology and the remaining $38.72 \%$ studied Sociology, both at the University of Buenos Aires.

Firstly a descriptive analysis of the items was performed. Secondly, the reliability of the scale was tested studding its internal consistence $(\alpha=.83)$. Simultaneously, the construct validity was test by an exploratory factor analysis and a confirmatory factor analysis. Finally, the validity of criteria was studied by analyzing the relations between the GBJW, RWA, SDO, and PI. The results suggest adequate psychometric properties of the GBJWS in this sample of university students and verified the unidimensionality of the scale $\left(\chi^{2}(\mathrm{df})=54,01_{(14)} ; \mathrm{S}-\mathrm{B} \chi^{2}\right.$ (df) $=41,77_{(14)}$; $\mathrm{S}-\mathrm{B} \chi^{2} /(\mathrm{df})=2,98 ; \mathrm{NNFI}=.93$; CFI $=.96$; $\Delta_{2}=.96 ;$ RMSEA $=.08$ ). Furthermore, GBJWS was positively associated with RWA $(r=.35$; $p<.01)$, SDO $(r=.36 ; p<.01)$ and negatively with PI $(r=-.37 ; p<.01)$. It is considered that this paper contributes to the measuring of the GBJW since presents an adaptation and validation of a scale that wasn't available in Spanish. This study had been done with a university sample because the construction and following adaptations of the original scales were made in such population (Bègue, 2002; Dalbert, Lipkus, Sallay, \& Goch, 2001; O Connor, Morrison, T. \& Morrison, M., 1996). The relations between political conservatism are coherent with the support of the status quo, since if the world is thought as a just place, there is no reason to transform it (Furnham, 2003; Jost \& Hunyady, 2005). This study had been done with a university sample because the construction and following adaptations of the original scales were made in such population (Bègue, 2002; Dalbert, Lipkus, Sallay \& Goch, 2001; O'Connor, Morrison, T. \& Morrison, M., 1996). However, it's necessary to point out that this limitation doesn't allow the generalization of the results to a general population. Hence, it is recommended for future studies to consider other populations to improve the generalization and representatively of the results pre- sented in this paper.

Key words: Lipkus; Global Belief in a Just World Scale; Argentina; Validation; Political ideology self positioning; RWA; SDO; University students.

\section{INTRODUCCIÓN}

Las investigaciones sobre la creencia en un mundo justo (Belief in a Just World - en adelante BJW) tienen su origen en los estudios realizados por Lerner $(1965,1980$; Lerner \& Clayton, 2011; Lerner \& Simmons, 
1966), quien puso de manifiesto que, como las situaciones de injusticia resultan amenazantes para los individuos, éstos necesitan creer que en la vida todos obtienen lo que merecen, para mantener una sensación de control sobre el medio. Si las personas no creyeran que las cosas ocurren como consecuencia de los actos realizados, se sentirían expuestas a todo tipo de situaciones desagradables que, si bien hoy le ocurren a otro, mañana podrían ocurrirle a ellos. De esta manera, para evitar la angustia que genera tal amenaza, culpabilizan a las víctimas de injusticias haciéndolas responsables de sus padecimientos. Durante más de 50 años, distintos estudios realizados en contextos diferentes (Barreiro, 2013; Furnham, 2003; Hafer \& Bègue 2005; Loo, 2002) han confirmado los hallazgos de Lerner (1965).

En las últimas décadas, se ha puesto de manifiesto que la BJW se encuentra presente en individuos de diferentes culturas, siendo evidentes sus relaciones con factores sociopolíticos (Furnham, 1993, 1998, 2003). Por ejemplo, en las sociedades con una mayor distancia de poder (Hofstede, 1984), aquellos que tienen más riquezas y poder presentan mayores niveles de BJW. Por el contrario, quienes tienen menos riquezas y acceso a recursos sociales, creen en mayor medida que el mundo es injusto. Asimismo, la BJW sostenida por los individuos pertenecientes a sectores sociales favorecidos condena y devalúa a los pobres, permitiéndoles lidiar con los sentimientos de culpa y justificando las numerosas injusticias sociales (Barreiro, 2008, 2009; Barreiro \& Castorina, 2005, 2006; Furnham, 2003; Zubieta \& Barreiro, 2006). De acuerdo a la teoría de la justificación del sistema (Jost, Federico \& Napier, 2009), no solo los grupos con una mejor posición económica sostienen y defienden el sistema social, económico y político en el que viven, también los grupos en una posición desfavorable necesitan justificarlo para sostener su autoestima (Jost \& Burgess, 2000). De esta manera, muchas personas que viven bajo sistemas sociales injustos y opresivos piensan que éstos son imperfectos, pero mejores que cualquier otra alternativa, aun- que resulten perjudicados (Jost \& Hunyady, 2005). Desde esta perspectiva, la BJW opera como una ideología justificadora del sistema, desempeñando una función paliativa de las injusticias al racionalizar el status quo, reduciendo la ansiedad, la culpa y la incertidumbre.

En este sentido, la BJW junto con la orientación a la dominancia social (Social Dominance Orientation, en adelante SDO) justifica las relaciones jerárquicas constitutivas de los sistemas económicos injustos (Jost \& Hunyady, 2005; Jost, Federico \& Napier, 2009; Liviatan \& Jost, 2011). Específicamente, la teoría de la dominancia social (Pratto, Sidanius, Stallworth \& Malle, 1994) postula la existencia de un mecanismo psicológico basado en el deseo de establecer y mantener las jerarquías sociales, es decir, la subordinación de ciertos grupos percibidos como inferiores a aquellos considerados superiores (Pratto, Stallworth \& Sidanius, 1997; Sidanius \& Pratto, 1999; Sidanius, Pratto \& Rabinowitz, 1994; Sidanius, Levin, Liu \& Pratto, 2000).

Por lo tanto, la negación de las injusticias mediante la BJW presenta dos facetas complementarias (Furnham, 2003). Por un lado, a nivel individual, resulta salugénica para los individuos (Dalbert, 2010), ya que les permite planificar su futuro. Además, como generalmente las personas se piensan a sí mismas como buenas, cuando atraviesan una situación que les genera padecimiento, la consideran algo pasajero que tarde o temprano terminará de manera favorable (Maës, 1998). En este sentido, la BJW permite que las personas afronten su propia victimización, reduciendo las posibilidades de sufrir depresión y estrés (Dalbert, 2010). Por otro lado, tiene efectos negativos a nivel social, no solo porque legitima las desigualdades, sino que distintos estudios han mostrado sus relaciones positivas con el autoritarismo favoreciendo diferentes formas de intolerancia, prejuicio y discriminación (Connors \& Heaven, 1987; Dalbert \& Yamuchi, 2003; Moore, 2008; Rubin \& Peplau, 1975, 1973). Según Lambert, Burroughs y Nguyen (1999; Lambert, Burroughs \& Chasteen, 1998), el 
autoritarismo y la BJW conforman un sistema de creencias con consecuencias antisociales que opera devaluando y culpabilizando a las víctimas de injusticias. Más aún, la BJW contribuye a dar estabilidad al entorno, preservando a los individuos del riesgo que éste supone para las personas autoritarias. En esta línea, se han identificado asociaciones entre la BJW y el autoposicionamiento ideológico-político (en adelante PI), particularmente con el extremo de derechas, debido al acuerdo con las formas políticas tradicionales que ofrecen un marco prescriptivo acerca de lo correcto y lo incorrecto (Connors \& Heaven, 1987; Rubin \& Peplau, 1975; Schlenker, Chambers \& Le, 2012; Smith \& Green, 1984).

Si bien el estudio de la BJW clásicamente se basó en situaciones experimentales, desde la construcción de Escala de Creencias en un Mundo Justo (Rubin \& Peplau, 1973, 1975) ha tenido primacía la indagación sistemática de las diferencias individuales en el constructo, dejando de lado otro tipo de abordajes (Lerner, 2003). No obstante, la amplia utilización de esta escala tuvo como consecuencia que se pongan en discusión diferentes aspectos de la misma (Furnham, 2003; Loo, 2002). Por una parte, se destacan las críticas recibidas a su estructura unidimensional, ya que diferentes estudios han reportado de dos a cinco factores, que indicarían su carácter multidimensional (e.g., Ambrosio \& Sheehan, 1990; Caputi, 1994; Fink \& Wilkins, 1976; Furnham \& Procter 1989; Loo 2002; Whatley, 1993). En este sentido, uno de los puntos más importantes en discusión es la relación entre la BJW y la creencia en un mundo injusto, dado que distintos estudios muestran que serían constructos independientes, aunque relacionados de manera negativa (Connors \& Heaven, 1987; Dalbert, 2010; Furnham, 1985; Furnham \& Procter, 1989; Loo, 2002). Por lo tanto, no se trataría de un único constructo. Del mismo modo, se ha señalado que las personas pueden pensar que el mundo es justo en un dominio (como el trabajo, la familia, etc.) pero no hacerlo con respecto a otros (Furnham \& Procter, 1989; Lipkus, 1991), así como tam- bién pueden considerar de manera diferente la BJW dependiendo de si se refieren al propio destino o al de otros (Dalbert, 2010; Dalbert, Lipkus, Sallay \& Goch, 2001; Lipkus, 1991). Por otra parte, distintos estudios han señalado que la consistencia interna de la escala de Rubin y Peplau $(1973,1975)$, evaluada con el coeficiente Alpha de Cronbach, suele ser baja (e.g., Couch, 1998; Lipkus, 1991; Loo, 2002; Mohr \& Luscri, 1995; Whatley, 1993).

En este marco Lipkus (1991) construyó una nueva escala con el objetivo de brindar una alternativa viable a la de Rubin y Peplau (1975) para la evaluación global de la BJW. La Escala de Creencia Global en un Mundo Justo (Global Belief in a Just World Scale GBJWS) está compuesta por 7 ítemes y fue testeada originalmente en una muestra de 402 estudiantes universitarios. El análisis factorial por máxima verosimilitud dio cuenta de un solo factor con un autovalor de 4.83, que explicó el $69 \%$ de la variancia total, mientras que la fiabilidad interna de la escala evaluada a través del estadístico Alpha de Cronbach indicó un valor de .82. De esta manera, la escala ha mostrado ser unidimensional y sus propiedades psicométricas han sido evaluadas en diferentes contextos como Francia $(\alpha=.86$; Bègue, 2002), Hungría ( $\alpha=.72$; Dalbert et al., 2001), Canadá ( $\alpha=.88$; O`Connor, Morrison T. \& Morrison, M., 1996) y Alemania $(\alpha=.65$; Dalbert et al., 2001).

El objetivo principal del estudio que se informa fue adaptar y validar la GBJWS (Lipkus, 1991) en una muestra de estudiantes universitarios de la Ciudad de Buenos Aires dado que no se cuenta con una versión en español de la misma. Para ello, en primer lugar, se realizó un análisis descriptivo de los ítemes. En segundo lugar, se analizó la consistencia interna de la escala y su validez de constructo. Finalmente, se estudió la validez de criterio de la escala mediante el análisis de sus relaciones con el RWA, la SDO y el PI.

\section{MÉTOdO}

El diseño del estudio fue de tipo instrumental (Montero \& Leon, 2007), ya que la 
finalidad del mismo era adaptar y validar la escala de GBJW (Lipkus, 1991) a una muestra de estudiantes universitarios de la Ciudad de Buenos Aires.

\section{PARTICIPANTES}

Participaron del estudio 328 estudiantes universitarios residentes en la Ciudad Autónoma de Buenos Aires, con edades comprendidas entre 18 y 40 años $(M=24.1$; $D T=3.88)$. El $36.6 \%$ eran hombres $(n=120)$ y el $63.4 \%$ mujeres $(n=208)$. El $61.28 \%$ eran alumnos de la Facultad de Psicología de la Universidad de Buenos Aires y el 38.72\% restante, de la Carrera de Sociología de la Facultad de Ciencias Sociales de la misma universidad. Además, el 5.79\% $(n=19)$ se autoposicionó como perteneciente a la clase social media-baja, el $83.23 \%$ $(n=273)$ a la clase media y el $10.97 \%$ $(n=36)$ a la clase media-alta.

Los sujetos participaron voluntaria y anónimamente luego de brindar su consentimiento. Asimismo, se les informó que los datos relevados serían utilizados con fines exclusivamente académico-científicos, bajo la Ley Nacional 25.326 de protección de datos personales. La administración de los instrumentos se realizó en un único encuentro.

\section{INSTRUMENTOS}

Se administaron las siguientes pruebas:

1.- Escala de Creencia Global en un Mundo Justo (GBJW - Lipkus, 1991): Se aplicó una versión adaptada de la escala original compuesta por siete ítemes (ver Tabla 1). El formato de respuesta es tipo Likert con cinco anclajes de respuesta desde 1: Totalmente en desacuerdo a 5: Totalmente de acuerdo. Mayores puntuaciones indican mayores niveles de creencia en un mundo justo.

2.- Escala de Autoritarismo del Ala de Derechas (RWA - Altemeyer, 1996): Para evaluar el constructo se utilizó una versión reducida de esta escala, adaptada y validada al contexto local (Etchezahar, 2012). Está compuesta por seis ítemes (e.g., "Nues- tro país necesita un líder poderoso que pueda enfrentar a los extremistas e inmorales que actualmente prevalecen en nuestra sociedad", "Hay muchas personas extremistas e inmorales tratando de arruinar las cosas; la sociedad debe detenerlos"). Para su validación se trabajó con tres muestras de 1.273 estudiantes universitarios de la Ciudad de Buenos Aires, cuya consistencia interna $(.73<\alpha<.83)$ y validez de constructo $(.98<\mathrm{CFI}<.99 ; .04$ $<$ RMSEA < .07) demostraron ser adecuadas. El formato de respuesta de la misma es tipo Likert con cinco anclajes de respuesta desde 1: Totalmente en desacuerdo a 5: Totalmente de acuerdo. Puntuaciones mayores indican mayores niveles de autoritarismo.

3.- Escala de Orientación a la Dominancia Social (SDO): Se administró una versión adaptada y validada al contexto argentino (Etchezahar \& Prado-Gascó, 2011) de la escala original (Pratto et al., 1994; Sidanius \& Pratto, 1999). En sus 10 ítemes se distinguen dos dimensiones del constructo: Dominancia grupal (e.g., "Para salir adelante en la vida, algunas veces es necesario pasar por encima de otros grupos de personas", "Todos los grupos superiores deberían dominar a los grupos inferiores") y Oposición a la igualdad (e.g., "Habría menos problemas si tratáramos a los diferentes grupos de manera más igualitaria", "Se debe aumentar la igualdad social") que en su conjunto conforman al constructo SDO. Las propiedades métricas de la escala fueron estudiadas en una muestra de 302 estudiantes universitarios de la Ciudad de Buenos Aires, cuya consistencia interna $(\alpha=.88)$ y validez de constructo $(\mathrm{CFI}=.94 ; \mathrm{RMSEA}=.07)$ demostraron ser adecuadas. El formato de respuesta sigue una escala de 1: Completamente en desacuerdo a 5: Completamente de acuerdo. Mayores niveles sugieren una mayor orientación a la dominancia social.

4.- Escala de Autoposicionamiento Ideológico-político (PI): Se utilizó una adaptación de la versión de Rodríguez, Sabucedo y Costa (1993), cuya consigna es "En asuntos de política la gente habla de izquierda y derecha, 
acorde con una escala de 1 a 5, siendo 1 de extrema derecha y 5 de extrema izquierda, ¿dónde se ubicaría usted?’.

5.- Variables sociodemográficas: Se diseñó un cuestionario ad-hoc para recabar este tipo de información. Entre las variables consideradas se encontraban: sexo, edad y clase social autopercibida.

\section{Procedimiento}

Para la adaptación al español de la GBJWS se siguieron los estándares metodológicos internacionales recomendados por la International Test Commission (ITC) (Hambleton, 1994, 2005). Se utilizó el método de traducción inversa (Muñiz \& Hambleton, 2000), llevado a cabo por dos intérpretes nativos de forma individual.

\section{ANÁLISIS DE LOS DATOS}

Para realizar el análisis estadístico de los datos se utilizaron los programas SPSS $21 \mathrm{y}$ EQS 6.2. En primer lugar, se calcularon los estadísticos descriptivos de los ítemes: media, desviación típica, asimetría y curtosis, de acuerdo con lo propuesto por Hair, Black, Babin, Anderson y Tatham (2006) en el marco de la Teoría Clásica de los Test. En segundo lugar se analizó la fiabilidad de la escala mediante el análisis de su consistencia interna utilizando el estadístico Alpha de Cronbach, considerando su variación si se elimina el elemento. Simultáneamente, se estudió la validez de constructo de la escala mediante el análisis factorial exploratorio y confirmatorio.

Finalmente, se evaluó la validez de criterio mediante el análisis de las relaciones de las puntuaciones en la escala GBJW con las obtenidas mediante las escalas RWA, SDO, PI.

\section{Resultados}

ANÁLISIS DE ITTEMES Y CONSISTENCIA INTERNA

Se analizaron los 7 ítemes que componen la escala original GBJWS (Lipkus, 1991). En la Tabla 1 se presenta la redacción final de los ítemes y para cada uno de ellos se informa: media $(M)$, desviación típica $(D T)$, correlación ítem-total $\left(r_{\mathrm{jx}}\right)$, Alpha de Cronbach si se elimina el elemento $(\alpha-x)$, asimetría $(S)$ y curtosis $(K)$.

En dicha tabla puede observarse que los niveles de asimetría y curtosis de las puntuaciones de todos los ítemes son adecuadas, considerándose como tales aquellas ubicadas en el intervalo \pm 2 (George \& Mallery, 2011). Asimismo, la consistencia interna de la escala $(\alpha=.83)$, examinada a través del estadístico Alpha de Cronbach, no mejora al eliminar alguno de los ítemes. La correlación ítem total indicó que todos los elementos contribuyen adecuadamente al conjunto de la escala $(r>.35$ - Hair et al., 2006).

\section{ANÁLISIS DE VALIDEZ DE CONSTRUCTO}

Tras analizar la fiabilidad de la escala se procedió a estudiar su validez de constructo mediante la realización de un análisis factorial exploratorio (AFE) seguido por un análisis factorial confirmatorio (AFC) dividiendo la muestra en dos para cada estudio.

En primer lugar se determinó la adecuación de los datos mediante la prueba KaiserMeyer-Olkin $(K M O=.823)$. El resultado del AFE, cuyo criterio de extracción de factores es el de considerar autovalores iguales o superiores a 1 , fue un modelo con una única dimensión que explicó el 50.19\% de la variancia.

En segundo lugar, para el cálculo del AFC se especificó un modelo unidimensional de acuerdo a la evidencia brindada por estudios previos (Bègue, 2002; Dalbert et al., 2001; O’Connor et al., 1996). Se utilizó como método de estimación el de máxima verosimilitud $(M L)$ con la corrección robusta de Satorra - Bentler (S-B - Bentler, 2006; Satorra, 2002), para controlar la posible incidencia de la no normalidad de los datos. Debido a la significatividad del estadístico chi cuadrado $(p<.01)$, en principio la adecuación de los datos al modelo teórico propuesto no se pudo asegurar. No obstante, dado que este estadístico es sensible al tamaño de la muestra, se tu- 
vieron en cuenta las sugerencias de Bentler (2006) y se recurrió a otros índices de ajuste, como el ratio entre el chi cuadrado y sus grados de libertad (considerando como aceptables valores por debajo de 5 - Hu \& Bentler, 1999), el Non Normed Fit Index (NNFI), el Comparative Fit Index (CFI) y el Bollen's Incremental Fit Index (IFI o $\Delta_{2}$ ) siendo indicadores de un buen ajuste los valores superiores a .90 (MacCallum \& Austin, 2000). Además se consideró el Root mean-square error of approximation (RMSEA), considerando a las puntuaciones menores a .08 como indicadoras de un ajuste apropiado (Browne \& Cudeck, 1992). Por último, se tuvieron en cuenta los coeficientes estandarizados $(\beta)$ de cada variable observada en la variable latente (Hair et al., 2006).

En la Figura 1 se presenta el modelo especificado con los indicadores de ajuste menciona$\operatorname{dos}\left(\chi_{(\mathrm{df})}^{2}=54,01_{(14)} ; \mathrm{S}-\mathrm{B} \chi_{(\mathrm{df})}^{2}=41,77_{(14)}\right.$; $\mathrm{S}-\mathrm{B} \chi^{2} /(\mathrm{df})=2,98 ; \mathrm{NNFI}=.93 ; \mathrm{CFI}=.96$; $\Delta_{2}=.96 ;$ RMSEA $\left.=.08\right)$.

\section{VALIDEZ DE CRITERIO}

Posteriormente, con el objetivo de incrementar la evidencia empírica de la validez de constructo, se analizó la validez de criterio de la GBJWS. Para ello se analizaron las relaciones entre las puntuaciones en la escala y RWA, SDO y PI, dado que distintos estudios han puesto de manifiesto la existencia de correlaciones positivas entre la BJW y el autoritarismo de derechas (Connors \& Heaven, 1987; Dalbert \& Yamuchi, 2003; Lambert et al., 1998; Lambert et al., 1999; Moore, 2008; Rubin \& Peplau, 1975, 1973), la orientación a la dominancia social (Bizer, Hart \& Jekogian, 2012) y el autoposicionamiento ideológico político de derechas (Connors \& Heaven, 1987; Rubin \& Peplau, 1975; Schlenker et al., 2012; Smith \& Green, 1984) (ver Tabla 2).

Como puede verse en la Tabla 2, todas las asociaciones con las variables testeadas fueron estadísticamente significativas. Positivas entre GBJWS, RWA y SDO y negativa con PI. Tales resultados son coherentes con los informados por los estudios previos que estudiaron la relación entre tales constructos, mencionados en el párrafo anterior, por lo tanto tales relaciones aportan un criterio de validez concurrente.

\section{Discusión}

El objetivo principal del estudio que se informa fue realizar la adaptación y validación de la GBJWS en una muestra de estudiantes universitarios de la Ciudad de Buenos Aires. Todos los ítemes que componen la escala GBJWS contribuyen a la evaluación del constructo. Asimismo, se analizó su consistencia interna obteniendo resultados adecuados y similares a los informados por estudios realizados en población de estudiantes universitarios de diferentes contextos como Francia (Bègue, 2002), Canadá (O`Connor et al., 1996) y Alemania (Dalbert et al., 2001). Paralelamente se analizó la validez de la GBJWS a través de un análisis factorial confirmatorio en el que se testeó el ajuste de los datos al modelo de siete ítemes agrupados en un único factor, presentando propiedades psicométricas adecuadas.

A continuación se analizó la validez de criterio de la GBJW analizando sus relaciones con RWA, SDO y PI. De esta manera, se identificó una correlación positiva entre los valores de la GBJWS y RWA, tal como señalan estudios previos (e.g., Connors \& Heaven, 1987; Lambert et al., 1999; Moore, 2008). En este sentido, puede pensarse que la negación de las injusticias por parte de la GBJWS resultaría adaptativa y funcional para salvaguardar a las personas autoritarias de la visión del mundo como un lugar peligroso y amenazante, tanto para sí mismos como para su grupo (Altemeyer, 1996; Etchezahar \& Prado-Gascó, 2012). De esta manera, el control sobre el entorno facilitado por la GBJW posibilitaría que las personas piensen que las desgracias que ocurren en la vida son la consecuencia de no haber seguido el 'camino correcto', culpabilizando a las víctimas y justificando así la agresión hacia quienes son considerados como diferentes, 
una característica distintiva de la personalidad autoritaria. Asimismo, la relación positiva entre GBJWS y SDO resulta coherente con los planteos que consideran que ambas justifican y racionalizan el sistema económico (Jost \& Hunyady, 2005). Según Phelan y Basow (2007), una mayor orientación a la dominancia social permite sostener la distancia social con aquellos individuos o grupos socialmente desfavorecidos, evitando un sentimiento de culpa por poseer mayor acceso a los recursos materiales. De este modo, dado que las diferencias individuales en SDO reflejan una motivación por la perpetuación de las relaciones intergrupales jerárquicas en la sociedad, la GBJWS justificaría tales diferencias apelando a los méritos individuales y negando así las injusticias sociales (Bizer, Hart \& Jekogian, 2012). En concordancia con trabajos previos (Ficarotto, 1990; Kurdek, 1988), se puso de manifiesto una asociación positiva entre la GBJWS y el autoposicionamiento ideológico político de derechas. Esta relación podría explicarse si se considera que, según la visión de las ideologías políticas de derecha, la distribución de la riqueza en la sociedad, las instituciones sociales y políticas, la división de roles, los sistemas financieros y de seguridad deben ser sostenidos para el desarrollo de la sociedad (Dittmar \& Dickinson, 1993). A su vez, su relación con el conservadurismo político resulta coherente con la pre- servación del status quo, dado que si el mundo es pensado como un lugar justo, no existen razones para intentar modificar la realidad a través de la acción política (Furnham, 2003; Jost \& Hunyady, 2005).

En esta línea, se sugiere la realización de estudios dedicados a analizar la GBJWS en el contexto argentino para profundizar el análisis de sus relaciones con el autoritarismo del ala de derechas y diferentes formas de prejuicio, ya que trabajos recientes proponen un modelo causal entre las variables mencionadas (ver Bizer et al., 2012).

Finalmente, el estudio realizado contribuye a la evaluación de la GBJW mediante la adaptación y la validación de un instrumento en español no disponible hasta el momento. No obstante, cabe señalar que este trabajo se llevó a cabo solo con una muestra de estudiantes universitarios, ya que la construcción de la GBJW y sus adaptaciones posteriores se realizaron con esa población (Bègue, 2002; Dalbert et al., 2001; O'Connor et al., 1996). Debido a dicha limitación, y a haber considerado solo estudiantes de la Ciudad de Buenos Aires, los resultados presentados no son generalizables a la población general. Por lo tanto, se recomienda llevar a cabo estudios que consideren diferentes poblaciones, para lograr una mayor generalización y representatividad de los resultados. 
TABLA 1

ANÁLISIS DESCRIPTIVO DE LOS ÍTEMES DE LA ESCALA DE GBJW

\begin{tabular}{|c|c|c|c|c|c|c|}
\hline Ítemes & $M$ & $D T$ & $r_{\mathrm{js}}$ & $\alpha-x$ & $S$ & $K$ \\
\hline $\begin{array}{l}\text { 1.- Creo que las personas obtienen lo que tienen } \\
\text { derecho a tener. }\end{array}$ & 230 & 122 & 63 & 80 & 58 & 75 \\
\hline $\begin{array}{l}\text { 2.- Creo que los esfuerzos de una persona son te- } \\
\text { nidos en cuenta y recompensados. }\end{array}$ & 2.98 & 1.27 & .46 & .83 & .09 & -1.12 \\
\hline $\begin{array}{l}\text { 3.- Creo que las personas se han ganado las re- } \\
\text { compensas y los castigos que reciben. }\end{array}$ & 2.43 & 1.28 & .70 & .79 & .51 & -.86 \\
\hline $\begin{array}{l}\text { 4.- Creo que las personas que tienen mala suerte } \\
\text { la han traído sobre sí mismas. }\end{array}$ & 2.01 & 1.09 & .50 & .82 & .77 & -.38 \\
\hline $\begin{array}{l}\text { 5.- Creo que las personas obtienen lo que mere- } \\
\text { cen. }\end{array}$ & 2.53 & 1.29 & .69 & .79 & .35 & -1.06 \\
\hline $\begin{array}{l}\text { 6.- Creo que las recompensas y los castigos son } \\
\text { administrados justamente. }\end{array}$ & 1.89 & 1.06 & .66 & .80 & 1.14 & .60 \\
\hline $\begin{array}{l}\text { 7.- Básicamente pienso que el mundo es un lugar } \\
\text { justo. }\end{array}$ & 1.82 & 1.01 & .42 & .83 & 1.04 & .30 \\
\hline
\end{tabular}


TABLA 2

CORRELACIONES ENTRE LA ESCALA DE GBJW Y VARIABLES RELACIONADAS

\begin{tabular}{|l|c|c|c|c|c|c|c|}
\hline & $\alpha$ & $M$ & $D T$ & 1 & 2 & 3 & 4 \\
\hline 1.- GBJWS & .83 & 2.48 & .85 & - & & & \\
2.- SDO & .81 & 1.97 & .65 & $.36^{* *}$ & - & & \\
3.- RWA & .82 & 2.85 & .95 & $.35^{* *}$ & $.29^{* *}$ & - & - \\
4.- PI & - & 3.34 & .75 & $-.37^{* *}$ & $-.32^{* *}$ & $-.30^{* *}$ & - \\
& & & & & & & \\
\hline
\end{tabular}

${ }^{* *} p<.01$

FIGURA 1

MOdelo UNIDIMENSIONAL DE LA ESCALA GBJW

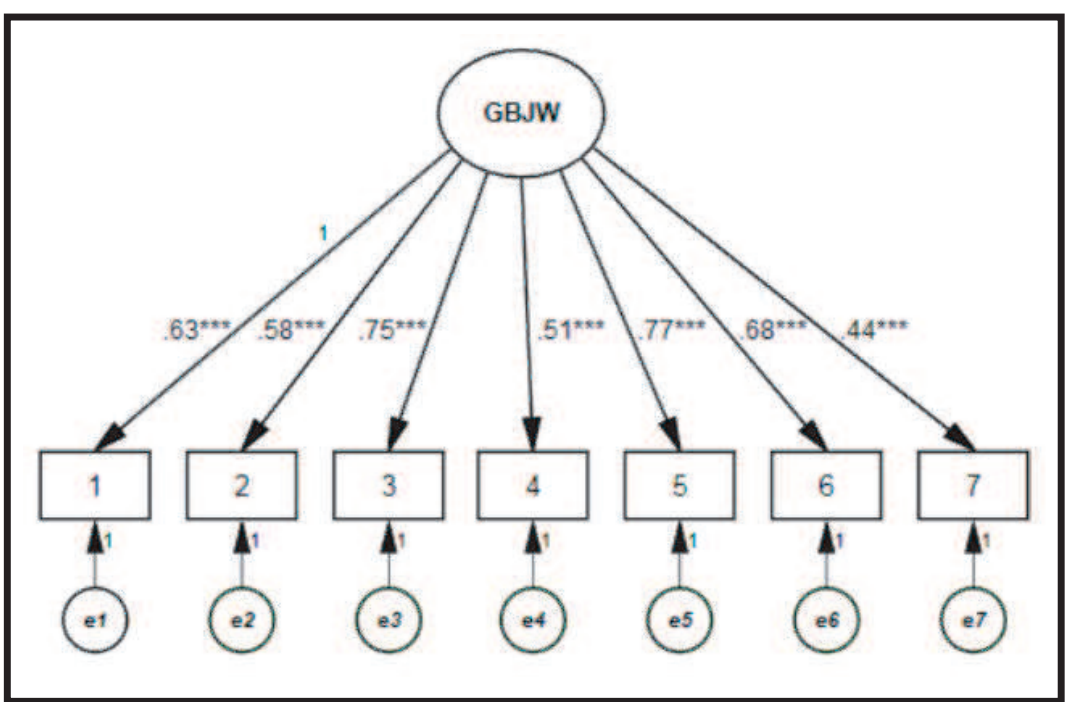

Nota:

Ajuste del modelo

$\chi^{2}(\mathrm{df})=54,01_{(14)}$

S-B $\chi^{2}(\mathrm{df})=41,77(14)$

$\mathrm{S}-\mathrm{B} \chi^{2 /}(\mathrm{df})=2,98$

$\mathrm{NNFI}=.93$

$\mathrm{CFI}=.96$

$\Delta_{2}=.96$

RMSEA $=.08$

${ }^{* * *} p<.001$ 


\section{REFERENCIAS BIBLIOGRÁFICAS}

Altemeyer, B. (1996). The authoritarian spectre. Cambridge: Harvard University Press.

Ambrosio, A. \& Sheehan, E. (1990). Factor analysis of the Just World Scale. The Journal of Social Psychology, 130(3), 413-415. http://dx.doi. org/10.1080/00224545.1990.9924600

Barreiro, A. (2008). El desarrollo de la creencia en un mundo justo: Relaciones entre la construcción individual del conocimiento y los saberes producidos colectivamente [Development of belief in a just world: Relations between individual construction of knowledge and knowledge collectively produced]. Estudios de Psicología, 29(3), 289-299. http://dx.doi.org/10. 1174/021093908786145403

Barreiro, A. (2009). La creencia en la justicia inmanente piagetiana: Un momento en el proceso de apropiación de la creencia ideológica en un mundo justo [The belief in Piaget immanent justice: A moment in the process of appropriation of the ideological belief in a just world]. Psykhe, 18(1), 73-84.

Barreiro, A. (2013). The appropriation process of the belief in a just world. Integrative Psychological and Behavioral Sciences, 47, 431-449.

Barreiro, A. \& Castorina, J.A. (2005). Las creencias en el mundo justo: ¿Un invariante cognitivo o una apropiación social? [Belief in a just world: Cognitive invariant or social appropriation?]. Psicologia da Educação, 21(2), 103-123.

Barreiro, A. \& Castorina, J.A. (2006). Dos perspectivas sobre la creencia en la justicia del mundo: Naturalismo versus legitimación ideológica [Two perspectives on belief in a just world: Naturalism versus ideological legitimation]. Espacios en Blanco, 16, 97-123.

Bègue, L. (2002). Beliefs in justice and faith in people: Just world, religiosity and interpersonal trust. Personality and Individual Differences,
32(3), 375-382. http://dx.doi.org/10.1016/S0 191-8869(00)00224-5

Bentler, P. (2006). EQS 6 structural equations program manual. Encino, CA: Multivariate Software, Inc.

Bizer, G.Y., Hart, J. \& Jekogian, A.M. (2012). Belief in a just world and social dominance orientation: Evidence for a mediational pathway predicting negative attitudes and discrimination against individuals with mental illness. Personality and Individual Differences, 52(3), 428-432. http://dx.doi.org/10.1016/j.p aid.2011.11.002

Browne, M. \& Cudeck, R. (1992). Alternative ways of assessing model fit. En K. Bollen \& J. Long (Eds.), Testing structural equation models (pp. 136-162). Beverly Hills, CA: Sage. http://dx.doi.org/10.1177/00491241920210020 05

Caputi, P. (1994). Factor structure of the just world scale among Australian undergraduates. Journal of Social Psychology, 134(4), 475-482. http://dx.doi.org/10.1080/00224545.1994.9712 198

Connors, J. \& Heaven, P.C. (1987). Authoritarianism and just world beliefs. Journal of Social Psychology, 127(3), 345-356. http://dx. doi.org/10.1080/00224545.1987.9713702

Couch, J.V. (1998). Another psychometric evaluation of the Just World Scale. Psychological Reports, 82(3), 1283-1286. http://dx.doi.org/ 10.2466/PR0.82.3.1283-1286

Dalbert, C. (2010). The justice motive as personal resource. Dealing with challenges and critical life events. New York: Kluwer Academic / Plenum.

Dalbert, C., Lipkus, I., Sallay, H. \& Goch, I. (2001). A just and an unjust world: Structure and validity of different world beliefs. Personality and Individual Differences, 30(4), 561577. http://dx.doi.org/10.1016/S0191-88 69(00) 00055-6 
Dalbert, C. \& Yamauchi, L.A. (1994). Belief in a just world and attitudes toward immigrants and foreign workers: a Cultural comparison between Hawaii and Germany. Journal of Applied Social Psychology, 24(18), 1612-1626. http://dx.doi.org/10.1111/j.1559-1816.1994.tb 01565.x

Dittmar, H. \& Dickinson, J. (1993). The perceived relationship between the belief in a just world and sociopolitical ideology. Social Justice Research, 6(3), 257-272. http://dx.doi.org/10. 1007/BF01054461

Etchezahar, E. (2012). Las dimensiones del autoritarismo: Análisis de la escala de autoritarismo del ala de derechas (RWA) en una muestra de estudiantes universitarios de la Ciudad de Buenos Aires [The authoritarianism dimmension: Analysis of right-wing authoritarianism scale (RWA) in a university student sample from Buenos Aires]. Revista Psicología Politica, 12(25), 591-603.

Etchezahar, E. \& Prado-Gascó, V. (2011). A preliminary model linking right and left-wing authoritarianism, social dominance and political ideology. Trabajo presentado en el 12th European Congress of Psychology, Turquía.

Etchezahar, E. \& Prado-Gascó, V. (2012). Autoritarisme de l'ala de dretes: Antecedents i perspectives de futur [Right-wing authoritarianism: past and future perspectives]. Anuari de Psicologia, 14(1), 147-157.

Ficarotto, T. (1990). Racism, sexism, and erotophobia: Attitudes of heterosexuals toward homosexuals. Journal of Homosexuality, 19 (1), 111-116. http://dx.doi.org/10.1300/J082v 19n01_07

Fink, H.C. \& Wilkins, W.E. (1976, abril). Belief in a just world, interpersonal trust, and attitudes. Trabajo presentado en la Eastern Psyhological Association, Kentucky.

Furnham, A. (1985). Just world beliefs in an unjust society: A cross cultural comparison. European
Journal of Social Psychology, 15(3), 363-366. http://dx.doi.org/10.1002/ejsp.2420150310

Furnham, A. (1993). Just world beliefs in twelve societies. The Journal of Social Psychology, 133(3), 317-329. http://dx.doi.org/10.1080/00 224545.1993.9712149

Furnham, A. (1998). Measuring beliefs in a just world. En L. Montada \& M. Lerner (Eds), Responses to victimizations and belief in the just world (pp. 141-162). New York: Plenum. http://dx.doi.org/10.1007/978-1-4757-6418-5_ 9

Furnham, A. (2003). Belief in a just world: Research progress over the past decade. Personality and Individual Differences, 34(5), 795817. http://dx.doi.org/10.1016/S0191-8869(0 2)00072-7

Furnham, A. \& Procter, E. (1989). Belief in a just world: Review and critique of the individual difference literature. British Journal of Social Psychology, 28(4), 365-384. http://dx.doi.or g/10.1111/j.2044-8309.1989.tb00880.x

George, D. \& Mallery, P. (2011). IBM SPSS Statistics 19 Step by Step: A Simple Guide and Reference. Boston: Pearson.

Hafer, C. \& Bègue, L. (2005). Experimental research on just-world theory: Problems, developments, and future challenges. Psychological Bulletin, 131(1), 128-167. http://dx. doi.org/10. 1037/0033-2909.131.1.128

Hair, J.F., Black, W.C., Babin, B.J., Anderson, R.E. \& Tatham, R.L. (2006). Multivariate data analysis. New Jersey: Pearson.

Hambleton, R. (1994). Guidelines for adapting educational and psychological tests: A progress report. European Journal of Psychological Assessment, 10, 229-244.

Hambleton, R. (2005). Issues, designs and technical guidelines for adapting test into multiple languages and cultures. En R. Hambleton, P. Merenda \& C. Spielberger (Eds.), Adapting educational and psychological tests for cross- 
cultural assessment (pp. 3-38). New Jersey: Lawrence Erlbaum.

Hofstede, G. (1984). The cultural relativity of the quality of life concept. Academy of Management Review, 9(3), 389-398. http://dx.doi.org/ 10.5465/AMR.1984.4279653

Hu, L.T. \& Bentler, P.M. (1999). Cutoff criteria for fit indexes in covariance structure analysis: Coventional criteria versus new alternatives. Structural Equation Modeling, 6(1), 1-55. http://dx.doi.org/10.1080/10705519909540118

Jost, J.T. \& Burgess, D. (2000). Attitudinal ambivalence and the conflict between group and system justification motives in low status groups. Personality and Social Psychology Bulletin, 26(3), 293-305. http://dx.doi.org/10. 1177/0146167200265003

Jost, J.T., Federico, C.M. \& Napier, J.L. (2009). Political ideology: Its structure, functions, and elective affinities. Annual Review of Psychology, 60(1), 307-333. http://dx.doi.org/10.114 6/annurev.psych.60.110707.163600

Jost, J.T. \& Hunyady, O. (2005). Antecedents and consequences of system-justifying ideologies. Current Directions in Psychological Science, 14(5), 260-265. http://dx.doi.org/10.1111/j.0 963-7214.2005.00377.x

Kurdek, L.A. (1988). Correlates of negative attitudes toward homosexuals in heterosexual college students. Sex Roles, 18(11-12), 727-738. http://dx.doi.org/10.1007/BF00288057

Lambert, A., Burroughs, T. \& Chasteen, A. (1998). Belief in a just world and right-wing authoritarianism as moderators of perceived risk. En L. Montada \& M. Lerner (Eds.), Responses to victimizations and belief in the just world (pp. 107-125). New York: Plenum. http://dx.doi. org/10.1007/978-1-4757-6418-5_7

Lambert, A., Burroughs, T. \& Nguyen, T. (1999). Perceptions of risk and the buffering hypothesis: The role of just world beliefs and rightwing authoritarianism. Personality and Social
Psychology Bulletin, 25(6), 643-656. http:// dx.doi.org/10.1177/0146167299025006001

Lerner, M.J. (1965). Evaluation of performance as a function of performer's reward and attractiveness. Journal of Personality and Social Psychology, 1(4), 355-360. http://dx.doi.org/10.1 037/h0021806

Lerner, M. (1980). The belief in a just world: a fundamental delusion. New York: Plenum.

Lerner, M. (2003). The justice motive: Where social psychologists found it, how they lost it, and why they may not find it again. Personality and Social Psychology Review, 7(4), 388-399. http://dx.doi.org/10.1207/S15327957PSPR070 4_10

Lerner, M. \& Clayton, S. (2011). Justice and selfinterest: Two fundamental motives. New York: Cambridge University Press.

Lerner, M.J. \& Simmons, C.H. (1966). The observer's reaction to the "innocent victim": Compassion or rejection? Journal of Personality and Social Psychology, 4(2), 203-210. http://dx.doi. org/10.1037/h0023562

Lipkus, I. (1991). The construction and preliminary validation of a global belief in a just worlds scale and the exploratory analysis of the multidimensional belief in a just world scale. Personality and Individual Differences, 12(11), 1171-1178. http://dx.doi.org/10.1016/0191-88 69(91)90081-L

Liviatan, I. \& Jost, J.T. (2011). System justification theory: Motivated social cognition in the service of the status quo. Social Cognition, 29(3), 231-237. http://dx.doi.org/10.1521/so co.2011. 29.3.231

Loo, R. (2002). Belief in a just world: Support for independent just world and unjust world dimensions. Personality and Individual Differences, 33(5), 703-711. http://dx.doi.org/10.1 016/S0191-8869(01)00185-4

MacCallum, R. \& Austin, J. (2000). Applications of structural equation modeling in psycholo- 
gical research. Annual Review of Psychology, 5l(1), 201-226. http://dx.doi.org/10.1146/an nurev.psych.51.1.201

Maës, J. (1998). Immanent justice and ultimate justice: Two ways of believing in justice. En L. Montada \& M.J. Lerner (Eds.), Responses to victimizations and belier in a just world (pp. 940). New York: Plenum.

Moore, D. (2008). Toward a more just world: What makes people participate in social action? En K.A. Hegtvedt \& J. Clay-Warner (Eds.), Justice (Advances in Group Processes, Volume 25) (pp. 213-239). Reino Unido: Emerald Group Publishing Limited. http://dx.doi. org/10.1016/ S0882-6145(08)25013-7

Mohr, P.B. \& Luscri, G. (1995). Social work orientation and just word beliefs. Journal of Social Psychology, 135(1), 101-103. http://dx. doi. org/10.1080/00224545.1995.9711408

Montero, I. \& León, O.G. (2007). A guide for naming research studies in Psychology. International Journal of Clinical and Health Psychology, 7(3), 847-862.

Muñiz, J. \& Hambleton, R. (2000). Adaptación de los tests de unas culturas a otras [Test adaptation from one culture to others]. Metodología de las Ciencias del Comportamiento, 2(2), 129-149.

O'Connor W., Morrison T. \& Morrison M. (1996). The reliability and factor structure of the global belief in a just world. Journal of Social Psychology, 136(5), 667-668. http://dx.doi.org/ 10.1080\%2F00224545.1996.9714055

Phelan, J.E. \& Basow, S.A. (2007). College students' attitudes toward mental illness: An examination of the stigma process. Journal of Applied Social Psychology, 37(12), 2877-2902. http://dx.doi.org/10.1111/j.1559-1816.2007.00 286.x

Pratto, F., Sidanius, J., Stallworth, L.M. \& Malle, B.F. (1994). Social dominance orientation: A personality variable predicting social and political attitudes. Journal of Personality and Social Psychology, 67(4), 741-763. http://dx. doi.org/10.1037//0022-3514.67.4.741

Pratto, F., Stallworth, L. \& Sidanius, J. (1997). The gender gap: Differences in political attitudes and social dominance orientation. British Journal of Social Psychology, 36(1), 49-68. http://dx.d oi.org/10.1111/j.2044-8309.1997.tb01118. x

Rodríguez, M., Sabucedo, J.M. \& Costa, M. (1993). Factores motivacionales y psicosociales asociados a distintos tipos de acción política [Motivational and psychosocial factors associated to different types of political action]. Psicología Politica, 7(2), 19-38.

Rubin, Z. \& Peplau, A. (1973). Belief in a just world and reactions to another's lot: a study of participants in the National Draft Lottery. Journal of Social Issues, 29(4), 73-93. http://dx.doi. org/10.1111/j.1540-4560.1973.tb00104.x

Rubin, Z. \& Peplau, L.A. (1975). Who believes in a just world? Journal of Social Issues, 31(3), 65-89. http://dx.doi.org/10.1111/j.1540-4560.1 975.tb00997.x

Satorra, A. (2002). Asymptotic robustness in multiple group linear-latent variable models. Econometric Theory, 18(2), 297-312. http://dx. doi.org/10.1017/S0266466602182041

Schlenker, B.R., Chambers, J.R. \& Le, B.M. (2012). Conservatives are happier than liberals, but why? Political ideology, personality, and life satisfaction. Journal of Research in Personality, 46(2), 127-146. http://dx.doi.or g/10. 1016/j.jrp.2011.12.009

Sidanius, J. \& Pratto, F. (1999). Social dominance: An intergroup theory of social hierarchy and oppression. Cambridge: University Press.

Sidanius, J., Pratto, F. \& Rabinowitz, J. (1994). Gender, ethnic status, ingroup attachment and social dominance orientation. Journal of CrossCultural Psychology, 25, 194-216.

Sidanius, J., Levin, S., Liu, J. \& Pratto, F. (2000). Social dominance orientation, anti-egalitar- 
ianism and the political psychology of gender: An extension and cross-cultural replication. European Journal of Social Psychology, 30(1), 41-67. http://dx.doi.org/10.1002/(SICI)1099-0 992(200001/02)30:1<41::AID-EJSP976>3.0.O ;2-O

Smith, K.B. \& Green, D.N. (1984). Individual correlates of the belief in a just world. Psychological Reports, 54(2), 435-38. http://dx. doi.org/10.2466/pr0.1984.54.2.435

Vila, N., Küster, I. \& Aldás, J. (2000). Desarrollo y validación de escalas de medida en marketing. Quaderns de Treball [Development and validation of Marketing scales. Workbooks]. Facultat d'Economia. Universitat de Valencia, 104.

Whatley, M. (1993). Belief in a just world: unidimensional or multidimensional? The Journal of Social Psychology, 133(4), 547-551. http:// dx.doi.org/10.1080/00224545.1993.9712180

Zubieta, E. \& Barreiro, A. (2006). Percepción social y creencia en el mundo justo. Un estudio con alumnos y estudiantes urbanos argentinos [Social perception and belief in a just world. A study whit Argentine students]. Revista de Psicología, 24(2), 175-178.

Instituto de Investigaciones Facultad de Psicología Universidad de Buenos Aires (UBA)

Fecha de recepción: 22 de julio de 2013 Fecha de aceptación: 12 de marzo de 2014 
